\title{
Optimal Net Energy and Foraging Behavior of Grus americana in Aransas National Wildlife Refuge*
}

Kristen Lawler ${ }^{\dagger}$ and Christian Schmidt $t^{\ddagger}$

${ }^{*}$ This research was conducted as part of a Research Experience for Undergraduates (REU) during the summer of 2016 at Sam Houston State University under the advisory of Drs. John Alford and Edward Swim

${ }^{\dagger}$ Department of Mathematics, Marist College, 3399 North Road, Poughkeepsie, NY 12601

${ }^{\ddagger}$ Department of Mathematics, The College of Saint Scholastica, 1200 Kenwood Avenue, Duluth, MN 55811 


\section{Abstract}

Whooping cranes (Grus americana) became endangered in the late 1800s when population numbers dwindled to as low as only 15 wild birds. Today, the wild population has significantly rebounded, but remains threatened. The last surviving migratory flock of whooping cranes travels to Texas every year for the winter. When the cranes arrive they primarily forage for wolfberries, but as the berry population decreases eventually the cranes' diet switches to one of mainly blue crabs. Understanding how abiotic conditions such as drought and water level fluctuations affect these food resources is important to wildlife managers who are tasked with the protection of the cranes. In this paper, we formulate a dynamic model that predicts the net energy intake of the whooping crane with parameters that may be used to control both the abiotic and biotic characteristics of the ecosystem inhabited during winter. We optimize the model to establish the maximum net energy intake of the crane over one season in ANWR and determine the point at which a crane will switch from foraging for berries to foraging for blue crab. This switching point gives insight to the cranes' foraging behavior, allowing us to have a better idea of what steps will be most effective moving forward if human action is needed for conservation of the species. Furthermore, the complete development and analysis within this paper will aid in determining whether or not sufficient resources exist in a crane's territory for it to sustain the winter and prepare for a successful flight back to breeding grounds.

\section{Introduction}

The Aransas-Wood Buffalo whooping crane population is the last surviving migratory flock of Whooping Cranes (Grus americana). They migrate from Wood Buffalo National Park (WBNP) in Alberta, Canada to the Aransas National Wildlife Refuge (ANWR) in Texas every autumn [3]. Whooping cranes became endangered during the late 1800s and the early 1900s due to overhunting and habitat loss, of which the latter remains a concern to this day [6]. Once the whooping cranes arrive in ANWR, generally in mid-October, they become fiercely territorial and thus each crane primarily hunts and forages within its own territory. Until their final departure in mid-March, the cranes will spend the majority of their time in the salt marshes of ANWR. In these marshes, they primarily forage for Carolina Wolfberries (Lycium carolinianum) in the vegetation and for blue crab (Callinectes sapidus) in the ponds [3].

The net energy accumulated while wintering in Texas plays a crucial role in the life history of the whooping cranes [8]. Recent droughts have caused drastic changes in the habitat of ANWR. Low water levels along with high salinity levels have affected the availability of both wolfberries and crabs, two primary food sources within the cranes' territories. In this paper, we formulate a mathematical model for predicting the net energy intake of the whooping crane. Our model is based on optimal foraging theory [12]. Analysis of this model yields the point in time at which the cranes change their primary prey preference from wolfberries to crabs. Knowledge of this switching point aids in our understanding of the cranes' prey dependency. Such an understanding is crucial not only to determining if human intervention is warranted, but also what that intervention should entail if deemed necessary. For example, 
considering the switching point with respect to the total time spent in ANWR, the remaining time in this territory after the switching point serves as an estimate of the time that cranes will be most reliant on blue crabs as a source of energy. This information could be used to help biologists better predict whether or not there is adequate crab availability to sustain the crane for the remaining period, thus determining whether human action is needed.

\section{Materials and Methods}

We used foraging theory [12] to develop a mathematical model that describes the net energy intake of the crane over one season in ANWR. Our model includes energy gain from the two primary food resources consumed by the crane in ANWR, wolfberries and blue crabs, and the energy loss due to hunting, foraging, traveling, and preening/resting. We then computed and verified a global maximum for net energy intake using the second derivative test. We compared this predicted maximum energy to that which is required for successful migration and an increased likelihood of reproductive success. To check for biological validity within our model we estimated all parameters, and finally completed a sensitivity analysis to identify which parameters are the most influential.

\section{Results}

\subsection{Formulation of the Mathematical Model}

We assume that specific prey is found within distinct "patches" of suitable habitat. In general, we let $T_{i_{j}}$ denote the time spent in a patch $j$ which contains prey type $i$. Let there be $k$ many patches of prey type $i$; then we denote the total time spent in all patches of type $i$ as $T_{i}=\sum_{j=1}^{k} T_{i_{j}}$. Let the average encounter rate, $\lambda_{i}$, be expressed as $\lambda_{i}=s \cdot d_{i}$ where $s$ is the area surveyed by the crane per unit time and $d_{i}$ is the population of prey type per unit area of patch type [9]. In general, we will assume $\lambda_{i}$ is time dependent since we expect that $d_{i}$ will itself depend on time. We can represent the total number of prey organisms consumed by a given crane as

$$
J=\sum_{i=1}^{N} T_{i} \lambda_{i} p_{i},
$$

where each $i=1, \ldots, N$ corresponds to one of $N$ total prey types that make up the crane's diet and $p_{i}$ denotes the crane's probability of successful capture upon encountering the prey. For simplicity, we assume $p_{i}$ constant.

When residing in ANWR, 94-97\% of the whooping cranes' diet consists of wolfberries and blue crabs [3]. Thus we will approximate total prey consumption using only two terms (where we index by letters $b$ and $c$ in place of 1 and 2) to get that

$$
J=T_{c} \lambda_{c} p_{c}+T_{b} \lambda_{b} p_{b}
$$

where prey type $c$ refers to crabs and $b$ refers to berries, respectively. Let $\alpha$ and $\beta$ denote the energy gain per crab and berry, respectively. Then equation (1) gives a formula for net 
energy, $\triangle A$ :

$$
\Delta A=\alpha\left(T_{c} \lambda_{c} p_{c}\right)+\beta\left(T_{b} \lambda_{b} p_{b}\right)-\phi
$$

Here, $\phi$ is used to denote the total energy loss of the crane (e.g. due to travel between patches as well as expenditures associated with hunting, foraging, and preening/resting). It is important to note that we assume the behaviors of hunting and foraging only occur within patches, and that when in a patch the only behavior modeled is hunting or foraging.

Since the encounter rates of the crane with its prey will change over time in response to prey availability, we developed a time-dependent function to describe $\lambda_{i}$ yielding a time-dependent model. Since the time scale of interest is only that which pertains to the time whooping cranes spend wintering in ANWR, we make the simplifying assumption that both the berry and crab availability, denoted $n_{b}$ and $n_{c}$ respectively, are affected mainly by crane foraging and flooding events. These quantities obey the following simple dynamics:

$$
\begin{gathered}
\frac{d n_{b}}{d t}=-f n_{b}, \\
\frac{d n_{c}}{d t}=-c n_{c}+a,
\end{gathered}
$$

Here $f$ and $c$ are positive constants describing the crane's rate of foraging for berries and hunting for crabs. The parameter $a$ is a constant rate describing an influx of crabs due to changing water inundation levels. Initially, we will assume that $a=0$. This assumption is biologically supported since whooping cranes have been observed hunting both on land and in water [3]. We therefore do not anticipate water level fluctuations to drastically alter prey availability or foraging behavior. The equations above are easily solved by separation of variables method to get

$$
\begin{gathered}
n_{b}=n_{b 0} e^{-f t}, \text { and } \\
n_{c}=n_{c 0} e^{-c t,}
\end{gathered}
$$

where $n_{b 0}$ and $n_{c 0}$ are the initial availabilities of berries and crabs, respectively, corresponding to the crane's arrival at ANWR. Using the relation $\lambda_{i}=s \cdot d_{i}$ and expressing $d_{i}$ in terms of $n_{b}$ and $n_{c}$ results in the following instantaneous encounter rates

$$
\begin{aligned}
& \lambda_{b}(t)=s \cdot \frac{n_{b 0} e^{-f t}}{h_{b}}, \\
& \lambda_{c}(t)=s \cdot \frac{n_{c 0} e^{-c t}}{h_{c}},
\end{aligned}
$$

where $h_{b}$ and $h_{c}$ are the total areas of all berry and crab patches, respectively. These encounter rates only pertain to time spent within a patch. Thus each $\lambda_{i}$ has a domain of $\left[0, T_{i}\right]$ and is maximized when $t=0$.

Equations (3) and (4) were substituted into equation (2) to get a time dependent expression for $\Delta A$. To determine the total energy accumulated during one season in ANWR, we integrated each term of $\Delta A$ over the time spent in each patch type, $T_{b}$ and $T_{c}$. The energy loss term, denoted $\phi$ in equation (2), was separated into loss pertaining to the time spent in patches of berries and crabs, $m_{b}$ and $m_{c}$, as well as an energy loss associated with the time 
spent between patches, $\ell$. This resulted in the following model for net energy intake of the whooping crane:

$$
A=\int_{0}^{T_{b}}\left(\beta \lambda_{b}(t) p_{b}-m_{b}\right) d t+\int_{0}^{T_{c}}\left(\alpha \lambda_{c}(t) p_{c}-m_{c}\right) d t-\int_{0}^{T-T_{b}-T_{c}} \ell d t
$$

where $T$ is the total time in ANWR. After integration, we therefore have that

$$
A=\frac{\varphi_{b}}{f}\left(1-e^{-f T_{b}}\right)-m_{b} T_{b}+\frac{\varphi_{c}}{c}\left(1-e^{-c T_{c}}\right)-m_{c} T_{c}-\ell\left(T-T_{b}-T_{c}\right),
$$

where

$$
\varphi_{b}=\frac{\beta s n_{b 0} p_{b}}{h_{b}} \text { and } \varphi_{c}=\frac{\alpha s n_{c 0} p_{c}}{h_{c}} .
$$

This mathematical description assumes that the cranes expend more energy while hunting crabs than they do foraging for berries. Furthermore, we would expect all foraging behavior to be associated with a higher energy expenditure than that of time spent resting, preening, or traveling, $\ell$. Definitions of all parameters in our net energy intake model, $A$, can be found in Table 1. It is important to note that all parameters have been assumed positive.

\subsection{Parameter Estimates}

Table 1: This table contains the definitions, units, and estimates of all parameters used in the net energy model equation (6).

\begin{tabular}{clcc}
\multicolumn{5}{c}{ Parameter Definition and Estimated Values } \\
\hline Parameter & \multicolumn{1}{c}{ Description } & Units & Estimate \\
\hline$\alpha$ & energy gain per Blue Crab & $\mathrm{kJ} / \mathrm{crab}$ & 139.4 \\
\hline$\beta$ & energy gain per wolfberry & $\mathrm{kJ} /$ berry & 1.3 \\
\hline$\gamma$ & total foraging time for berries and crabs & days & - \\
\hline$c$ & rate of crane predation of crabs & $\frac{1}{d a y}$ & 0.065 \\
\hline$f$ & rate of crane foraging of berries & $\frac{1}{d a y}$ & 0.87 \\
\hline$h_{b}$ & area of berry patches & $m^{2}$ & 878,800 \\
\hline$h_{c}$ & area of crab patches & $m^{2}$ & 310,900 \\
\hline$\ell$ & energy loss outside of foraging in patches & $\frac{k J}{d a y}$ & 2,016 \\
\hline$m_{b}$ & energy loss while foraging for berries & $\frac{k J}{d a y}$ & $3,001.6$ \\
\hline$m_{c}$ & energy loss while foraging for crabs & $\frac{k J}{d a y}$ & $3,225.6$ \\
\hline$n_{b 0}$ & number of initial berries & berries & $6,837,064$ \\
\hline$n_{c 0}$ & number of initial crabs & - & 739,182 \\
\hline$p_{b}$ & probability of successful foraging of berries & - & 0.36 \\
\hline$p_{c}$ & probability of successful capture of crabs & - & 0.01 \\
\hline$s$ & rate of area surveyed by crane while foraging & $\frac{m^{2}}{d a y}$ & $9,197.928$ \\
\hline$k_{b}$ & proportional change in $I_{b}$ & - & 0.2 \\
\hline$k_{c}$ & proportional change in $I_{c}$ & - & 20 \\
\hline
\end{tabular}


The parameters in Table 1 were estimated to reflect the Boat Ramp territory of ANWR. First, our values for $h_{b}$ and $h_{c}$ both came directly from Slack's description of the Boat Ramp territory [10]. Parameters $n_{b 0}$ and $n_{b 0}$ also came from Slack and his recorded berry and crab densities in the same territory. The probabilities of successful capture were estimated utilizing data from Chavez-Ramirez [3]. The rates of foraging and successful capture of crabs and wolfberries, calculated using probes per minute and successful probes upon capturing prey, were compared to extrapolate $p_{b}$ and $p_{c}$ [3]. The parameter $s$, which is the rate of area surveyed by the crane while foraging, was calculated by utilizing the average foraging speed of a whooping crane [5] and multiplying it by the area of a half-circle with the radius equal to the neck length of the crane, where we are assuming the neck is approximately $40 \%$ of the total crane height, approximately 1.5 meters [14]. Energy loss parameters, $m_{b}, m_{c}$, and $\ell$, were determined utilizing the basal metabolic rate (BMR) and energy expenditure coefficients (EEC) [3]. Finally, our energy conversion rates, $\alpha$ and $\beta$, as well as our rates of hunting and foraging, $c$ and $f$, were estimated based upon the work of Krapu and Chavez-Ramirez. We discuss the possible values for the parameter in the next section.

\subsection{Computing Maximum Net Energy Intake}

We now use the net energy equation (6) to determine $T_{b}$ and $T_{c}$ that maximize $A$ and derive bounds for the total hunting/foraging time $\gamma=T_{b}+T_{c}$. For this analysis, we consider all parameters to be constants and $A$ to be a function of both $T_{b}$ and $T_{c}$. Thus we will write $A$ as $A\left(T_{b}, T_{c}\right)$ and use standard analysis from multivariable calculus to determine the maximum of $A$. In order to find a possible maximum, we calculate the gradient of $A$ as

$$
\nabla A\left(T_{b}, T_{c}\right)=\left(\varphi_{b} e^{-f T_{b}}-m_{b}+\ell, \varphi_{c} e^{-c T_{c}}-m_{c}+\ell\right) .
$$

The components of the gradient vector have units of $\mathrm{kJ} /$ day and indicate the (average) daily rate at which energy is acquired by the crane due to consumption of berries and crabs. Thus, for example, if the initial number of berries per unit area $\left(\frac{n_{b 0}}{h_{b}}\right)$ increases, the crane will necessarily be able to increase its daily energy intake from berry consumption.

Setting $\nabla A=0$, we solved for $T_{b}$ and $T_{c}$. As a result, we have a possible extremum of

$$
\left(T_{b}^{*}, T_{c}^{*}\right)=\left(-\frac{1}{f} \cdot \ln \left(\frac{m_{b}-\ell}{\varphi_{b}}\right),-\frac{1}{c} \cdot \ln \left(\frac{m_{c}-\ell}{\varphi_{c}}\right)\right) .
$$

We assume that the cranes expend more energy while hunting crabs than they do foraging for berries and that foraging behavior is associated with a higher energy expenditure than that of time spent resting, preening, or traveling between patches. It is important to note that this final assumption's validity depends upon the dispersion of patches within a given territory. Together, these assumptions imply that the parameters $\ell, m_{b}$ and $m_{c}$ are ordered as $\ell<m_{b}<m_{c}$ and each of the logarithms in the expression for $\left(T_{b}^{*}, T_{c}^{*}\right)$ is defined. We also assume

$$
\varphi_{b} \geq m_{b}-\ell \text { and } \varphi_{c} \geq m_{c}-\ell
$$

which then implies that $T_{b}^{*} \geq 0$ and $T_{c}^{*} \geq 0$. Thus, here we have that the (average) daily rate at which energy is acquired by the crane due to consumption of berries and crabs is 
greater than the energy lost while foraging for berries and crabs (respectively) minus energy expenditure due to time spent resting, preening, or traveling between patches.

After computing second derivatives, it can be verified using equation (6) that the following inequalities hold:

$$
\frac{\partial^{2} A}{\partial T_{b}^{2}}<0 \quad \text { and } \quad \frac{\partial^{2} A}{\partial T_{b}^{2}} \cdot \frac{\partial^{2} A}{\partial T_{c}^{2}}-\left(\frac{\partial^{2} A}{\partial T_{b} \partial T_{c}}\right)^{2}>0,
$$

where each second derivative is evaluated at $\left(T_{b}^{*}, T_{c}^{*}\right)$ given in (7). Thus, by use of the Second Derivative Test, $A$ has a local maximum at $\left(T_{b}^{*}, T_{c}^{*}\right)$. To determine if $A\left(T_{b}^{*}, T_{c}^{*}\right)$ is a global maximum, we must evaluate $A\left(T_{b}^{*}, T_{c}^{*}\right)$ on the boundaries of the domain of $A$ given by

(i) $T_{c}=0$

(ii) $T_{b}=0$ and

(iii) $T_{b}+T_{c}=\gamma$,

where $\gamma$ is the total time spent foraging for prey so that $0 \leq T_{c} \leq \gamma$ and $0 \leq T_{b} \leq \gamma$. We denote the net energy evaluated on the $k^{t h}$ boundary as $A(k)=A_{k}$ and derive conditions for which $A\left(T_{b}^{*}, T_{c}^{*}\right)>A_{k}, k=i, i i, i i i$ which guarantees $A\left(T_{b}^{*}, T_{c}^{*}\right)$ is a global maximum for $A$.

On boundary (i), $T_{c}=0$ and

$$
A_{i}=\frac{\varphi_{b}}{f}\left(1-e^{-f T_{b}}\right)-m_{b} T_{b}-\ell\left(T-T_{b}\right)
$$

Therefore

$$
\frac{d A_{i}}{d T_{b}}=\varphi_{b} e^{-f T_{b}}-m_{b}+\ell
$$

so that $A_{i}$ is maximized when

$$
T_{b}=T_{b}^{*}=-\frac{1}{f} \cdot \ln \left(\frac{m_{b}-\ell}{\varphi_{b}}\right)
$$

and

$$
\max \left\{A_{i}\right\}=\frac{\varphi_{b}}{f}\left(1-\frac{m_{b}-\ell}{\varphi_{b}}\right)+\frac{m_{b}-\ell}{f} \ln \left(\frac{m_{b}-\ell}{\varphi_{b}}\right)-\ell T .
$$

On boundary (ii), $T_{b}=0$ and

$$
A_{i i}=\frac{\varphi_{c}}{c}\left(1-e^{-c T_{c}}\right)-m_{c} T_{c}-\ell\left(T-T_{c}\right) .
$$

Therefore,

$$
\frac{d A_{i i}}{d T_{c}}=\varphi_{c} e^{-c T_{c}}-m_{c}+\ell
$$

so that $A_{i i}$ is maximized when

$$
T_{c}=T_{c}^{*}=-\frac{1}{c} \cdot \ln \left(\frac{m_{c}-\ell}{\varphi_{c}}\right)
$$


and

$$
\max \left\{A_{i i}\right\}=\frac{\varphi_{c}}{c}\left(1-\frac{m_{c}-\ell}{\varphi_{c}}\right)+\frac{m_{c}-\ell}{c} \ln \left(\frac{m_{c}-\ell}{\varphi_{c}}\right)-\ell T .
$$

After substituting (7) into the expression for $A$ in (6) we get that

$A\left(T_{b}^{*}, T_{c}^{*}\right)=\frac{\varphi_{b}}{f}\left(1-\frac{m_{b}-\ell}{\varphi_{b}}\right)+\frac{\varphi_{c}}{c}\left(1-\frac{m_{c}-\ell}{\varphi_{c}}\right)+\frac{m_{c}-\ell}{c} \ln \left(\frac{m_{c}-\ell}{\varphi_{c}}\right)+\frac{m_{b}-\ell}{f} \ln \left(\frac{m_{b}-\ell}{\varphi_{b}}\right)-\ell T$.

Note that $A\left(T_{b}^{*}, T_{c}^{*}\right)=\max \left\{A_{i}\right\}+\max \left\{A_{i i}\right\}+\ell T$ and $A\left(T_{b}^{*}, T_{c}^{*}\right)>\max \left\{A_{i}\right\}$ precisely when

$$
\varphi_{c}\left(1-\frac{m_{c}-\ell}{\varphi_{c}}\right)+\left(m_{c}-\ell\right) \ln \left(\frac{m_{c}-\ell}{\varphi_{c}}\right)>0
$$

which can be rewritten as

$$
\frac{\varphi_{c}}{m_{c}-\ell}-\ln \left(\frac{\varphi_{c}}{m_{c}-\ell}\right)>1,
$$

and similarly, $A\left(T_{b}^{*}, T_{c}^{*}\right)>\max \left\{A_{i i}\right\}$ precisely when

$$
\frac{\varphi_{b}}{m_{b}-\ell}-\ln \left(\frac{\varphi_{b}}{m_{b}-\ell}\right)>1
$$

Note that based upon our prior assumptions regarding parameter values seen on page 5 $\left(\varphi_{b} \geq m_{b}-\ell\right.$ and $\left.\varphi_{c} \geq m_{c}-\ell\right)$ inequalities (8) and (9) always hold.

We next derive an upper bound on $\gamma$ so that $A\left(T_{b}^{*}, T_{c}^{*}\right)>\max \left\{A_{i i i}\right\}$. Equivalently, since on boundary (iii) we have $T_{c}=-T_{b}+\gamma$, we show that $A\left(T_{b}^{*}, T_{c}^{*}\right)>A_{i i i}\left(T_{b}\right)$ for $0 \leq T_{b} \leq \gamma$ where

$$
A_{i i i}\left(T_{b}\right)=\frac{\varphi_{b}}{f}\left(1-e^{-f T_{b}}\right)+\frac{\varphi_{c}}{c}\left(1-e^{-c\left(-T_{b}+\gamma\right)}\right)+T_{b}\left(m_{c}-m_{b}\right)-\gamma m_{c}-\ell(T-\gamma) .
$$

Note that since $0 \leq T_{b}$, it then follows that $A_{i i i}\left(T_{b}\right)<B\left(T_{b}\right)$ where

$$
B\left(T_{b}\right)=\frac{\varphi_{b}}{f}+\frac{\varphi_{c}}{c}\left(1-e^{-c\left(-T_{b}+\gamma\right)}\right)+T_{b}\left(m_{c}-m_{b}\right)-\gamma m_{c}-\ell(T-\gamma) .
$$

The function $B$ is easily differentiated to get $\frac{d B}{d t}=-\varphi_{c} e^{-c\left(-T_{b}+\gamma\right)}+m_{c}-m_{b}$ which will be negative when $-\varphi_{c} e^{-c \gamma}+m_{c}-m_{b}<0$ or

$$
\gamma<-\frac{1}{c} \cdot \ln \left(\frac{m_{c}-m_{b}}{\varphi_{b}}\right)
$$

If this inequality holds then $B$ is decreasing everywhere and $B\left(T_{b}\right) \leq B(0)$. This implies that $A_{i i i}\left(T_{b}\right)<B(0)$ where

$$
B(0)=\frac{\varphi_{b}}{f}+\frac{\varphi_{c}}{c}\left(1-e^{-c \gamma}\right)-\gamma m_{c}-\ell(T-\gamma)
$$

Therefore, a sufficient condition so that $A\left(T_{b}^{*}, T_{c}^{*}\right)>A_{i i i}\left(T_{b}\right)$ is that $A\left(T_{b}^{*}, T_{c}^{*}\right)>B(0)$. Utilizing the parameters in Table 1 we find that $\varphi_{b} \approx 33490 \mathrm{~kJ}$ and $\varphi_{c} \approx 30485 \mathrm{~kJ}$ and, after substituting these values in $(7)$, we get $T_{b}^{*} \approx 4.1$ days and $T_{c}^{*} \approx 49.7$ days. Then the net energy from equation (6) is $A\left(T_{b}^{*}, T_{c}^{*}\right) \approx 119,288 \mathrm{~kJ}$. The left side of (8) is 22 and the left side of (9) is 30 so that both of the inequalities (8) and (9) are satisfied which guarantees that $A\left(T_{b}^{*}, T_{c}^{*}\right)$ is greater than both $\max \left\{A_{i}\right\}$ and $\max \left\{A_{i i}\right\}$. The inequality (10) is approximately $\gamma<77.04$ days. If $\gamma=77$ days, we get from $(11)$ that $B(0) \approx 106,795 \mathrm{~kJ}$ and it follows that $A\left(T_{b}^{*}, T_{c}^{*}\right)>B(0)$ and $A\left(T_{b}^{*}, T_{c}^{*}\right)$ is greater than $\max \left\{A_{i i i}\right\}$. 


\subsection{Maximal Net Energy Sensitivity Analysis}

To determine which parameters most heavily affect net energy intake, we completed a relative sensitivity analysis [7]. This was accomplished in the following manner: let $\delta$ be a parameter and let $F$ be an equation whose sensitivity to we want to check. Then the relative sensitivity of $F$ to $\delta$ is

$$
\frac{\partial F}{\partial \delta} \cdot \frac{\delta}{F} .
$$

The resulting sensitivities for our net energy model allowed us to rank our parameters from highest to lowest in regard to how heavily they impact net energy intake at our global maximum $A\left(T_{b}^{*}, T_{c}^{*}\right)$. The results are displayed in Table 2 where we use $M$ to denote $\left(T_{b}^{*}, T_{c}^{*}\right)$. For example, the first row of Table 2 is obtained by evaluating

$$
\frac{\partial A\left(T_{b}^{*}, T_{c}^{*}\right)}{\partial h_{c}} \cdot \frac{h_{c}}{A\left(T_{b}^{*}, T_{c}^{*}\right)} .
$$

Negative outputs reflect an inverse relationship whereas positive outputs show a direct relationship between the parameter and $A\left(T_{b}^{*}, T_{c}^{*}\right)$. We did not test the sensitivities of the parameters $\alpha, \beta$, and $s$ as these parameters are assumed to be constants.

Inspection of Table 2 shows that the parameters that impact the maximum energy intake the most are $h_{c}, n_{c}, p_{c}$, and $c$. For example, we see from the value displayed in row 1 that if we increase the area of the crab patches $\left(h_{c}\right)$ by a certain amount, there will be a corresponding $378 \%$ decrease (as this value is negative) in $A\left(T_{b}^{*}, T_{c}^{*}\right)$. This means that changes in the crab population would have the most drastic impact regarding the survivability of the whooping cranes as a species.

Table 2: Contains the units, sensitivity and ranking of all parameters used in the sensitivity analysis of the global maximum of $A$.

\begin{tabular}{clcc}
\hline \multicolumn{5}{c}{ Parameter Sensitivity } & & \\
\hline Parameter Tested & \multicolumn{1}{c}{ Sensitivity } & Value & Rank \\
\hline$h_{c}$ & $-\frac{\alpha s n_{c} p_{c}}{h_{c}^{2}}\left(\frac{e^{-c T_{c}}}{-c}+\frac{1}{c}\right) \cdot \frac{h_{c}}{A(M)}$ & -3.7756 & 1 \\
\hline$n_{c}$ & $\frac{\alpha p_{c}}{h_{c}}\left(\frac{e^{-c T_{c}}}{-c}+\frac{1}{c}\right) \cdot \frac{n_{c}}{A(M)}$ & 3.7756 & 1 \\
\hline$p_{c}$ & $\frac{\alpha n_{c}}{h_{c}}\left(\frac{e^{-c T_{c}}}{-c}+\frac{1}{c}\right) \cdot \frac{p_{c}}{A(M)}$ & 3.7756 & 1 \\
\hline$c$ & $\frac{\alpha s n_{c} p_{c}}{h_{c}}\left(\frac{e^{-c T_{c}}}{c^{2}}+\frac{T_{c} e^{-c T_{c}}}{c}-\frac{1}{c^{2}}\right) \cdot \frac{c}{A(M)}$ & -3.2722 & 2 \\
\hline$\left(-151+T_{b}+T-c\right) \cdot \frac{\ell}{A(M)}$ & -1.6444 & 3 \\
\hline$\ell$ & $\left(-\frac{m_{c}}{A(M)}\right.$ & -1.3424 & 4 \\
\hline$m_{c}$ & $\left(-T_{c}\right) \cdot \frac{m_{c}}{A\left(M n_{b}\right.}$ & -0.3132 & 5 \\
\hline$h_{b}$ & $-\frac{\beta s p_{b}}{h_{b}^{2}}\left(\frac{e^{-f T_{b}}}{-f}+\frac{1}{f}\right) \cdot \frac{h_{b}}{A(M)}$ & 0.3132 & 5 \\
\hline$n_{b}$ & $\frac{\beta s p_{b}}{h_{b}}\left(\frac{e^{-f T_{b}}}{-f}+\frac{1}{f}\right) \cdot \frac{n_{b}}{A(M)}$ & 0.3132 & 5 \\
\hline$p_{b}$ & $\frac{\beta n_{b}}{h_{b}}\left(\frac{e^{-f T_{b}}}{-f}+\frac{1}{f}\right) \cdot \frac{p_{b}}{A(M)}$ & -0.2797 & 6 \\
\hline$f$ & $\frac{\beta s n_{b} p_{b}}{h_{b}}\left(\frac{e^{-f T_{b}}}{f^{2}}+\frac{T_{b} e^{-f T_{b}}}{f}-\frac{1}{f^{2}}\right) \cdot \frac{f}{A(M)}$ & -0.1020 & 7 \\
\hline$m_{b}$ & $\left(-T_{b}\right) \cdot \frac{m_{b}}{A(M)}$ & &
\end{tabular}




\subsection{Finding a Switching Point}

Our model predicts the times that should be spent in each patch type, that is $\left(T_{b}^{*}, T_{c}^{*}\right)$ from (7), in order to maximize net energy. It does not, however, explain how these times are allocated throughout the entire wintering period in ANWR. To gain insight on these allotments, we turn to the switching point analysis.

To identify a switching point after the initial arrival of the cranes based purely upon prey preference, we must compare the two preys' energy efficiency ratios [9]. The point in time that satisfies equality of these ratios is our switching point, following the methods of H.R. Pulliam. The energy efficiency ratios for crabs and berries are defined as follows:

$$
\frac{\beta}{I_{b}} \text { and } \frac{\alpha}{I_{c}} \text {. }
$$

Each numerator is defined to be the energy intake per prey, and each denominator is defined as the time involved with the prey [9]. We define involvement times, $I_{i}$, to be inversely proportional to the encounter rates:

$$
I_{b}=\frac{k_{b}}{\lambda_{b}} \text { and } I_{c}=\frac{k_{c}}{\lambda_{c}},
$$

where $k_{b}$ and $k_{c}$ are positive constants of proportionality. Substituting the involvement times into the energy efficiency ratios, using the expressions in (3) and (4) for $\lambda_{b}$ and $\lambda_{c}$, and setting these equal yields

$$
\frac{\beta n_{b 0} e^{-f t}}{k_{b} h_{b}}=\frac{\alpha n_{c 0} e^{-c t}}{k_{c} h_{c}} .
$$

Solving for $t$ gives us a switching point at

$$
t_{s}=\frac{1}{f-c} \cdot \ln \frac{k_{c} h_{c} \beta s n_{b 0}}{k_{b} h_{b} \alpha s n_{c 0}} .
$$

The switching point, $t_{s}$, must be defined and non-negative, and so our parameters must satisfy the following conditions: if $0<k_{b} h_{b} \alpha s n_{c 0}<k_{c} h_{c} \beta s n_{b 0}$, then we must have

$$
f-c>0,
$$

and if $k_{b} h_{b} \alpha s n_{c 0}>k_{c} h_{c} \beta s n_{b 0}$, then we must have

$$
f-c<0 .
$$

Based upon our estimated parameters from Table 1, condition (12) is met. However, we do not assume that this will be the case across all territories within ANWR. Thus we note both conditions here, (12) and (13), so as to consider various biological possibilities, and also to mathematically ensure we are not left with a negative $t_{s}$. Utilizing our estimates, we then have an initial switch in prey preference from berries to crabs after approximately 96 hours of accumulated foraging. Hence, after about 10.66 days in ANWR, the optimal strategy is predicted to switch from foraging for berries to hunting for blue crab. This was calculated considering the total number of hours a day we would anticipate a whooping crane 
would spend foraging/hunting throughout the winter. We believe this calculation to be fairly reasonable, albeit slightly under the anticipated amount of time indicated by observational data: about 2-3 weeks. However, since this is considered to be our initial switch in prey preference, the time discrepancy between our calculations and field data may be attributed to the existence of a later, more final switch of preference.

\subsection{Switching Point Sensitivity Analysis}

In order to determine which parameters have the greatest effect on our switching point, $t_{s}$, we again completed a relative sensitivity analysis. Similar to the sensitivity analysis pertaining to net energy, parameters that were not tested include $\alpha, \beta$, and $s$. Also, $m_{b}$, $m_{c}$, and $\ell$ were disregarded in our analysis since $t_{s}$ does not depend on these parameters. The ranking of parameter sensitivity for $t_{s}$ was found to be essentially identical across all parameters except that of $f,-0.395$, and $c, 0.0295$. The other relevant parameters had a value of 0.328 with sign mirroring that of Table 2 . Interpreting what this means, if we consider the area of berry patches for example, this suggests that if we increase $h_{b}$ by a given amount, there will be a corresponding $32.8 \%$ increase in $t_{s}$. An overall analysis of these sensitivity values indicates that the switching point is minimally affected by the rate of hunting for crabs, and is most sensitive to the rate of foraging for berries. Biologically, this makes sense since the wolfberries are observed to be the primary food source upon the cranes' arrival. Thus the point in time at which a switch in this initial preference is made would be heavily influenced by the rate at which the available berries are consumed.

\section{Discussion}

In this paper we have formulated a mathematical model for the net energy intake, given by $A$ in equation (6), of the whooping crane population which overwinters in the Aransas National Wildlife Refuge (ANWR). Our model was created by considering and analyzing the primary sources of energy intake, blue crabs and wolfberries, as well as energy expenditure for various activities of the crane. In order to consider both biotic and abiotic factors within this model, we included not only the availability, but also the dispersion of the energy sources throughout the crane's territory. To estimate the resulting parameters from such considerations, we primarily referred to the field observations of Chavez-Ramirez, Cronin, Hartup, and Krapu for parameters related to crane behavior and energy intake, and Slack and Butzler for estimates related to the prey [8],[3],[5],[6].

The net energy intake, $A$ (from equation 6 ), is important to wildlife managers in ANWR. This quantity plays a primary role in the crane's ability to survive and reproduce after winter has concluded. When male and female cranes are able to gain approximately $20 \%$ and $16 \%$ of their body weight in fat during the winter, respectively, it is expected that sufficient conditions have been met not only to successfully migrate to their breeding grounds, but also to increase the probability of successful reproduction [8]. Thus, averaging these respective percentages gives us an idea of how much any randomly selected crane should gain, regardless of its sex, in order for us to anticipate survival, 18\%. If $W$ represents a crane's weight in grams [8], then the net energy required for a crane to survive and reproduce after winter has 
concluded, denoted $E$ relates to the crane's weight in the following way:

$$
E=0.18 W(39)=7.02 W
$$

since $0.18 \mathrm{~W}$ represents the average weight that must be gained by a crane, and 39 is the conversion of a gram of fat to energy, kJ [3]. Therefore, when using our model for analysis, a higher probability of reproductive success will be anticipated when $A \geq 7.02 \mathrm{~W}$.

Friends of the Wild Whoopers state the average hatching rate of a whooping crane egg to be $48 \%$ [11]. Analyzing data for the hatching rate of eggs after winters where there was a surplus of available food resources (based upon the above inequality), we were able to conclude that the probability of successful hatching given that the net energy intake is at least $7.02 \mathrm{~W}$ (as noted before, $\mathrm{W}$ represents the weight of the crane in grams):

$$
P(\text { Successful Hatching } \mid A \geq 7.02 W)=0.823
$$

or $82.3 \%$, which shows a substantial growth in successful hatching in years following food and energy surplus in ANWR [8].

Our mathematical analysis showed that the net energy intake $A$ is maximized when the total time spent hunting for berries $\left(T_{b}\right)$ and crabs $\left(T_{c}\right)$ is given by $\left(T_{b}^{*}, T_{c}^{*}\right)$ from $(7)$. In our analysis, we assumed that there is an upper bound on $\gamma=T_{c}+T_{b}$ from (10) and, using our parameters from Table 1, this bound becomes $\gamma<77.04$ days. This is consistent with the findings of Chavez-Ramirez [3]. It suggests that no more than $76.975 \%$ of daylight hours are spent hunting or foraging, coinciding with his estimated time allotment of 60-80\% [3]. At maximum energy, when $T_{b}^{*} \approx 4.1$ days and $T_{c}^{*} \approx 49.7$, we computed that a whooping crane spends approximately 8.5347 hours a day hunting and foraging. This was calculated by summing together $T_{b}^{*}$ and $T_{c}^{*}$ to find a total time hunting and foraging. This time was then multiplied by 24 to convert to hours and divided by 151 days, the total number of days that a crane typically spends in ANWR. Based upon the behavioral time allotments in [3], we believe this to be a reasonable prediction. Assuming that the average weight of a crane is $7 \mathrm{~kg}$, we have that the anticipated energy requirement, $E$, is approximately 49,140 kJ [3]. Finally, our calculation of a maximum net energy of $A\left(T_{b}^{*}, T_{c}^{*}\right) \approx 119,288 \mathrm{~kJ}$ is large relative to $E$, and so we expect a high probability of successful reproduction for a crane within this territory.

Our sensitivity analysis allowed us to make conclusions with both a mathematical and biological significance. It revealed that the parameters with the largest impact on maximum energy intake are $h_{c}, n_{c}, p_{c}$, and $c$, all of which pertain to crabs and the crane's interactions with them. This suggests that crabs are a more vital part of the cranes' diet; thus their availability and dispersion should be looked to as a key indicator of habitat quality and should be heavily considered in conservation efforts.

Some of the parameters that impact the maximum energy the least are those parameters that pertain to berries and the crane's interactions with them: $h_{b}, n_{b}, p_{b}$, and $f$. Biologically, this makes sense since the cranes spend most of their time hunting for and gain most of their energy from, the blue crabs. Knowing which parameters impact energy intake the most is crucial in determining what steps should be taken to guarantee a higher net energy intake of the whooping crane. 
In addition to developing a model for net energy intake, we also utilized optimal foraging theory to explain the observed behavior of the cranes initially foraging for berries and then switching their primary focus to hunting crabs [13]. When the cranes first arrive, the time spent in either patch is zero and thus both encounter rates are maximal. Convenience in terms of location is therefore initially of larger importance regarding prey preference [12]. This accounts for the cranes' choice to first forage for wolfberries. If the wolfberries' energy efficiency ratio is greater than that of the blue crabs, we would anticipate that the cranes would forage for berries. Similarly, if the crabs' energy efficiency ratio is greater than that of the wolfberries, we would expect the cranes to show a preference towards hunting crabs. Therefore, the time that satisfies equality of these ratios is anticipated to be the point in time at which the crane's prey preference would initially switch from one to the other, and more specifically in this case, from berries to crabs.

As the encounter rate with prey changes over time, we would expect that the crane's time involved with the prey, regarding search and handling, would change as well. In our energy efficiency ratios, we determined that this time involved with prey should therefore be inversely related to the encounter rates. This inverse relationship accounts for changes in the search and handling time per prey in response to changes in prey availability. It also accounts for a learning curve as a result of gained experience with the prey. Since the only time dependent component of our energy efficiency ratios is the encounter rates, we have that the cranes will have a switch in prey preference when their encounter rate with berries has decreased to the appropriate proportion of the crab encounter rate.

After finding our switching point, $t_{s}$, we determined several mathematical conditions that must be met. These conditions are also of biological importance. Requirements (12) and (13) essentially mean that if the rate of energy intake per day for berries is greater than that of crabs, then the foraging rate for berries should be greater than the hunting rate for crabs and vice versa. Since whooping cranes are optimal foragers, this is assumed to be satisfied.

Using our parameter estimates, we found a switching point after approximately 90.96 hours of accumulated foraging. This coincides with our maximal value for $T_{b}$ since based on the recorded behavior, we would expect most of the foraging for berries to occur when the cranes first arrive, followed by a switch in behavior towards primarily hunting for crabs. After the initial switch in prey preference, data reflects that some occasional foraging for berries does occur, but it pales in comparison to that of the crabs [3]. This is represented by the value of $T_{b}-t_{s}$. Based purely upon foraging theory, however, $T_{b}-t_{s}>0$ suggests that other minor switches in prey preference may occur later on.

To improve the predictive capabilities of our modeling efforts, several changes could be made to allow for better connections with data that is collected in the field. For example, we would like to initially expand upon the probabilities of successful capture. Here, we have assumed them to be constant; however, a more accurate depiction of these probabilities would include a distribution. Furthermore, to consider and analyze the impact of water inundation levels on prey availability we would like to consider the possibility of $a$ being nonzero. In doing so, a would become time dependent and periodic reflecting water level fluctuations associated with tidal changes. By combining field data for water levels, foraging success rates, and other observations at ANWR with components of our model, predictions for food resource availability for the cranes may be assisted by application of our energy analysis and verified through examination of hatching rates. Furthermore, in addition to the 
whooping cranes, we anticipate that through minimal alterations, this model could serve as a resource availability predictor for other threatened or endangered species as well. As a result, this model could be the foundation for future species conservation research: a vital topic to consider with the rapidly changing climate conditions being experienced worldwide. In fact, incorporating more environmental factors such as changing water inundation levels would extend our model to not only be a predictor of resource availability, but also a predictor of impacts from climate and environmental changes. 


\section{References}

[1] Butler, M. J., Harris, G., \& Strobel, B. N. (2013). Influence of whooping crane population dynamics on its recovery and management. Biological Conservation, 162, 89-99. doi:10.1016/j.biocon.2013.04.003

[2] Butzler, R. E. (2006). Growth patterns of Carolina wolfberry (Lycium carolinianum L.) in the salt marshes of Aransas National Wildlife Refuge, Texas, USA (Unpublished master's thesis). Texas A\&M University.

[3] Chavez-Ramirez, F. (1996). Food availability, foraging ecology, and energetics of Whooping Cranes wintering in Texas (Unpublished doctoral dissertation). Texas A\&M University.

[4] Chitnis, N., Hyman, J. M., \& Cushing, J. M. (2008). Determining Important Parameters in the Spread of Malaria Through the Sensitivity Analysis of a Mathematical Model. Bulletin of Mathematical Biology Bull. Math. Biol., 70(5), 1272-1296. doi:10.1007/s11538-008-9299-0

[5] Cronin, T. W., Kinloch, M. R., \& Olsen, G. H. (2005). Head-bobbing behavior in foraging whooping cranes favors visual fixation. Current Biology, 15(7). doi:10.1016/j.cub.2005.03.036

[6] Hartup, B. K., Olsen, G. H., \& Czekala, N. M. (2005). Fecal corticoid monitoring in whooping cranes (Grus americana) undergoing reintroduction. Zoo Biology Zoo Biol., 24(1), 15-28. doi:10.1002/zoo.20033

[7] John G. Alford, Alacia Voth, Edward Swim, Mathematical Modeling of Continuous and Intermittent Androgen Suppression for the Treatment of Advanced Prostate Cancer, Mathematical Biosciences and Engineering, Volume 14, Number 3, June 2017, pp. 777-804, doi:10.3934/mbe.2017043.

[8] Krapu, G. L., Iverson, G. C., \& Reinecke, K. J. (1985). Fat Deposition and Usage by Arctic-Nesting Sandhill Cranes during Spring. The Auk, 102(2), 362-368. doi: $10.2307 / 4086780$

[9] Pulliam, H. R. (1974). On the Theory of Optimal Diets. The American Naturalist, 108(959), 59-74. doi:10.1086/282885

[10] Slack, R. D. (2009). Linking freshwater inflows and marsh community dynamics in San Antonio Bay to whooping cranes. College Station, TX: Texas A \& M AgriLIFE.

[11] Stehn, T. (2002, February). Whooping Crane Recovery Activities: September, 2001 February, 2002.

[12] Stephens, D. W., \& Krebs, J. R. (1986). Foraging theory. Princeton, NJ: Princeton University Press. 
[13] Westwood CM, Chavez-Ramirez F (2005) Patterns of food use of wintering whooping cranes in coastal Texas. Proceedings North American Crane Workshop 9:133-140

[14] "Whooping Crane (Grus Americana)." TPW. Texas Parks and Wildlife Foundation, 2002. Web. 26 July 2016. 